\title{
No news without new scientific ideas
}

\author{
Anton J. M. Loonen*
}

Pharmacotherapy of Psychiatric Patients, Department of Pharmacy, University of Groningen, Groningen, The Netherlands

In this editorial, it is strongly advocated that a change of policy is warranted in order to prevent neuroscience from becoming a waste of time and money in the 21 st century. Repeating the same trick in different patient populations and perusing the scientific literature seems to currently be the backbone of medical science. However, this approach does not provide knowledge on how the brain works or how specific dysfunctions result in specific diseases. Therefore, earlier findings should, first, be combined to develop new theories on the mechanics of the mind, and, second, these new ideas should be tested in well-designed experiments.

Received 27 August 2013; Accepted 5 October 2013; First published online 13 December 2013

Key words: art, bankruptcy of neuroscience, philosophy, theory of knowledge, von Bertalanffy.

A well-known phenomenon in radiology is unexpected malignancies being overlooked on routine X-rays. My father had an X-ray of his chest in June 1982 that, retrospectively, clearly showed a bronchus tumor, which only became symptomatic in September 1982 and caused his death in May of the following year. When you do not know what to look for, even the most obvious aberrations are easily missed. This limitation of diagnostic capabilities is not a human flaw, but is intricately connected to the theory of knowledge. Scientific findings cannot be interpreted without the proper prerequisites, ie, a model to explain the background of the observations.

The theory of knowledge specifies at least 2 different strategies for finding a representation of the truth. ${ }^{1,2}$ The first strategy is to deduce a model from complex observations that can correctly predict the occurrence of a phenomenon. The second approach is formed by an empirical cycle; observations lead, by a process termed "induction," to a theory that results from deducing the development of a hypothesis. This hypothesis is either confirmed or falsified by new observations, which should lead to adaptation of the theory. The necessity of following these rules in order to create something new makes science an art form that is driven by human intelligence.

*Address for correspondence: Prof. Anton J. M. Loonen, MD, PharmD, PhD, Pharmacotherapy and Pharmaceutical Care, Department of Pharmacy, University of Groningen, Antonius Deusinglaan 1, 9713AV Groningen, The Netherlands.

(Email a.j.m.loonen@rug.nl)
However, disappointment is unavoidable when considering many of the scientific papers currently being published in medical journals. Many studies do not start with something similar to the above procedure. Unfortunately, the authors of many papers seem to have more interest in repeating a trick applied in an earlier paper. The most important aspect of doing science seems to be having your name in a prominent place on a paper published in one of the top-10 journals in a field. Since the advent of evidence-based medicine, a tremendous need has emerged to repeat randomized, controlled comparisons of all types of treatments. Thousands of patients are tested according to strict protocols in order to calculate characteristics, such as the "number needed to treat." During this process, the results of many separate studies are combined in a meta-analysis.

The same is now true for investigations of the mechanisms that underlie certain mental disorders, such as neuroimaging studies to elucidate the mechanism underlying depression. This research started with an estimation of the brain structures that are more or less active in significantly depressed patients. Studies then followed to elucidate the correct sequence and magnitude of activation. In May 2013, 3888 citations existed in PubMed for fMRI studies of patients with depression, 340 of which were clinical studies and 456 review articles. Forty-three of the review articles claimed to have followed a systematic approach. This strategy of perusing the scientific literature is very useful for distinguishing between methodological artifacts and true findings. However, this approach provides little 
information about how the brain works and how depression occurs. In the 1950 s to the 1970 s, psychoanalytically oriented psychotherapists could describe how the mind works in great detail and how various types of mental disorders occur. Unfortunately, the therapists had no evidence to support their theories. Today, the situation is reversed; we have an abundance of data about a number of specified physiological, electronic, and biochemical processes and substances within the brains of normal and sick people, but nobody dares to reflect on how the brain works or how mental disorders occur. Such papers will probably not be accepted due to a lack of supporting evidence.

In this issue of CNS Spectrums, Alan Pehrson and Connie Sanchez summarize evidence that supports an interaction between the serotonergic and glutamatergic neurotransmitter systems that provides relief from depression. ${ }^{3}$ The observation that intravenous administration of the noncompetitive N-methyl-D-aspartate (NMDA) receptor antagonist ketamine induces an almost immediate mood change in depressed patients was an important starting point for the search for interactions that possibly explain the antidepressant effects of existing and new "serotonergic" drugs. 4 However, glutamate is one of the major neurotransmitters of the central nervous system. Approximately $30 \%$ of CNS neurons and half of CNS synapses are glutamatergic. Therefore, we have to wonder where ketamine's site of action is located. Is it within the amygdala, the hippocampal complex, the basal ganglia, the brain stem, or elsewhere? Perhaps the interaction between the glutamatergic and serotonergic systems can help us to develop a theory on the mechanism of action of the mood-relieving effects of ketamine. Only such a theory can help us develop new effective treatment strategies.

Everything within the brain is connected to every other part, and everything seems to influence every other part. This complexity is how the brain originates in the embryonic stage. ${ }^{5}$ First, a structure forms from glial cells, and then neuronal stem cells migrate to their final positions in, for example, the cerebral cortex. After generating a critical mass of neurons, fibers start to develop by connecting all possible parts of the CNS. Most connections disappear again in subsequent years, leaving networks with a suitable function. Thus, the brain is a chaotic tangle of networks.

Since Karl Ludwig von Bertalanffy developed his General Systems Theory at the end of the $1940 \mathrm{~s},{ }^{6}$ scientists have known that they can only study the general functioning of a structure at a certain system level by going to a higher system level and finding out how the subsystems mutually relate to one another. For example, to determine how different brain structures interact in depression, the circuits within the brain should be described first. Identification of the higher level circuit makes possible a description of the interaction between its components on a lower system level, and this information could explain what goes wrong in depression. The same may be true in interpreting genetic data. Biochemical processes with an essential function in the healthy mood state should be identified first, and then the essential or rate-limiting steps within this chain of biochemical activities can be identified. Next, the genes that control these steps can be identified and polymorphisms that may alter the activity of these genes sought out. Genome-wide association studies are probably fruitless because most mental illnesses are unlikely to be due to inborn errors of metabolism caused by single genetic defects. Headless searching for an association is not useful.

In my opinion, medical science has had many undesirable developments in the 21 st century. We should appreciate the general rules of the philosophy of science again. Nothing new will happen in science without new ideas, theories, models, and hypotheses that we can test for validity and drop when we falsify them.

\section{Disclosure}

Anton Loonen does not have any disclosures.

\section{REFERENCES:}

1. De Groot AD. Methodologie. 's-Gravenhage, the Netherlands: Uitgeverij Mouton; 1961.

2. Loonen AJM, Zwanikken GJ. Farmaca, psychofarmaca, klinisch onderzoek. 's-Hertogenbosch, the Netherlands: Uitgeverij Wulco; 1987.

3. Pehrson AL, Sanchez C. Serotonergic modulation of glutamate neurotransmission as a strategy for treating depression and cognitive dysfunction. CNS Spectr. 2013.

4. Covvey JR, Crawford AN, Lowe DK. Intravenous ketamine for treatment-resistant major depressive disorder. Ann Pharmacother. 2012; 46(1): 117-123.

5. O'Rahilly R, Müller F. The Embryonic Human Brain, 3rd ed. Hoboken, NJ: John Wiley \& Sons, Inc.; 2006.

6. von Bertalanffy KL. An outline of general system theory. British Journal for the Philosophy of Science. 1950; 1(2): 134-165. 